\title{
Determination of psychosis-related clinical profiles in children with autism spectrum disorders using latent class analysis
}

\author{
Marinos Kyriakopoulos • Argyris Stringaris • Sofia Manolesou • \\ Maja Drobnič Radobuljac • Brian Jacobs • Avi Reichenberg • \\ Daniel Stahl $\cdot$ Emily Simonoff $\cdot$ Sophia Frangou
}

Received: 26 March 2014/ Accepted: 9 June 2014 / Published online: 26 June 2014

(c) Springer-Verlag Berlin Heidelberg 2014

\begin{abstract}
In children with autism spectrum disorders (ASD), high rates of idiosyncratic fears and anxiety reactions and thought disorder are thought to increase the risk of psychosis. The critical next step is to identify whether combinations of these symptoms can be used to categorise individual patients into ASD subclasses, and to test their relevance to psychosis. All patients with ASD $(n=84)$ admitted to a specialist national inpatient unit from 2003 to 2012 were rated for the presence or absence of impairment in affective regulation and anxiety (peculiar phobias, panic episodes, explosive reactions to anxiety), social deficits (social disinterest, avoidance or withdrawal and abnormal attachment) and thought disorder (disorganised or illogical thinking, bizarre fantasies, overvalued or delusional ideas). Latent class analysis of individual symptoms was conducted to identify ASD classes. External validation of these
\end{abstract}

M. Kyriakopoulos · B. Jacobs · E. Simonoff

Child and Adolescent Mental Health Clinical Academic Group,

South London and the Maudsley NHS Foundation Trust,

London, UK

M. Kyriakopoulos $(\bowtie) \cdot$ A. Stringaris · B. Jacobs ·

A. Reichenberg · D. Stahl - E. Simonoff

Department of Child and Adolescent Psychiatry, Institute of

Psychiatry, King's College London, P066, De Crespigny Park, London SE5 8AF, UK

e-mail: marinos.kyriakopoulos@kcl.ac.uk

M. Kyriakopoulos · A. Reichenberg · S. Frangou

Icahn School of Medicine at Mount Sinai, New York, USA

S. Manolesou

Central and North West London NHS Foundation Trust, London, UK

M. D. Radobuljac

University Psychiatric Hospital, Ljubljana, Slovenia classes was performed using as a criterion the presence of hallucinations. Latent class analysis identified two distinct classes. Bizarre fears and anxiety reactions and thought disorder symptoms differentiated ASD patients into those with psychotic features (ASD-P: $51 \%$ ) and those without (ASD-NonP: $49 \%$ ). Hallucinations were present in $26 \%$ of the ASD-P class but only $2.4 \%$ of the ASD-NonP. Both the ASD-P and the ASD-NonP class benefited from inpatient treatment although inpatient stay was prolonged in the ASD-P class. This study provides the first empirically derived classification of ASD in relation to psychosis based on three underlying symptom dimensions, anxiety, social deficits and thought disorder. These results can be further developed by testing the reproducibility and prognostic value of the identified classes.

Keywords Psychotic symptoms - MCDD - Autism spectrum disorder - Inpatient

\section{Introduction}

Autistic and psychotic disorders have historically been considered as related diagnostic entities. In the late 1970s, autism spectrum disorders (ASD) and schizophrenia (SZ) were split into two different diagnostic categories (DSMIII). The boundaries between the two disorders, however, continue to be debated. ASD and SZ share common neurobiological processes [1, 2] and genetic risk factors [35]. In addition, the presence of autistic traits in early childhood increases the risk of psychotic experiences in adolecence [6] and of SZ and SZ spectrum disorders in adulthood [7]. Conversely, a significant proportion of adults with SZ also fulfils criteria of childhood ASD [8]. Better characterisation of psychosis-related symptom 
profiles in children with ASD is likely to advance our understanding of these overlaps.

A potentially fruitful approach is to improve the clinical charaterisation of children with ASD that present with psychotic experiences. There is some evidence that affective symptoms in children with ASD may mediate the emergence of psychotic symptoms. In particular, formal thought disorder in ASD has been found to be induced by anxiety and stress [9]. This concept is also supported by reports of higher rates of psychotic symptoms in ASD children with significant symptoms of anxiety and throught disorder. It has been suggested that these children may represent a particular subgroup of ASD refered to as multiple complex developmental disorder (MCDD) [1012]. The criteria for MCDD are shown in Table 1 and highlight the importance of peculiar and idiosyncratic fears, panic episodes and explosive emotional behaviours [10]. Although anxiety symptoms were noted in the original description of classic autism [13] they do not feature as core symptoms of autism in more recent operational descriptions of the disorder. However, large-scale epidemiological studies (e.g. [14] ) and a recent meta-analysis [15] estimated that approximately $40 \%$ of ASD children have a comorbid anxiety disorder. A comprehensive review by White and colleagues [16] also reported that ASD children experiencing anxiety are more likely to show explosive behaviours in response to their fears and phobias. Weisbrot and colleagues [17] examined the relationship between specific types of anxiety and psychotic experiences in 483 children with ASD. The 6- to 12-year olds with ASD whose parents and teachers considered as highly anxious were also more likely to receive more severe ratings of psychotic symptoms (hallucinations, odd thoughts, bizzare behaviours) compared to those with with low levels of anxiety. The association with psychoticism was more evident for children who reported peculiar fears and phobias. Therefore, the MCDD criteria regarding affect regulation may be useful in capturing the symptom profiles of ASD children most likely to experience psychosis.

The MCDD criteria also highlight thought disorder as a dimension of psychoticism. More recent studies have suggested that thought disorder is a feature of ASD that relates more closely to the degree of language abnormalities rather than to SZ or spectrum disorders $[9,18]$. However, the study by Weisbrot and colleagues [17] demonstrated a link between odd thoughts, strange beliefs and illogical thinking (considered as symptoms of thought disorder according to MCDD criteria), in highly anxious ASD children who also experienced hallucinations. However, thought disorder, characterised by odd thinking, is a symptom domain that is similar between patients at high risk for SZ and ASD children meeting diagnostic criteria for MCDD [19]. In addition, thought disorder is a symptom domain that is highly predictive of conversion to psychosis in those at high risk regardless of developmental history [20]. It therefore appears important to consider the presence of thought disorder when attempting to define clinical profiles of ASD children likely to present with psychotic features.

The evidence summarised above suggests that the concept of MCDD is highly relevant to psychosis. At the same time, the argument for MCDD as a separate diagnostic entity has not received widespread support [4] and is not included in the latest, fifth version of the Diagnostic and Statistical Manual of Mental Disorders (DSM-5) or the planned revision of the International Classification of Diseases (ICD). Our interest in the MCDD does not relate to whether or not it constitutes an independent diagnostic

Table 1 Multiple complex developmental disorder research criteria

(1) Impaired regulation of affective state and anxieties

a. Unusual or peculiar fears and phobias or frequent idiosyncratic or bizarre anxiety reactions

b. Recurrent panic episodes or flooding with anxiety

c. Episodes of behavioural disorganisation punctuated by markedly immature, primitive or violent behaviours

(2) Impaired social behaviour

a. Social disinterest, detachment, avoidance or withdrawal despite evident competence

b. Markedly disturbed and/or ambivalent attachments

(3) The presence of thought disorder

a. Irrationality, magical thinking, sudden intrusions on normal thought process, bizarre ideas, neologism or repetition of nonsense words

b. Perplexity and easy confusability

c. Overvalued ideas including fantasies of omnipotence, paranoid preoccupations, over-engagement with fantasy figures, referential ideation

Diagnostic rules

A diagnosis of MCDD can be made if an individual meets total of five (or more) criteria from (1), (2), and (3) with at least one item from (1), one item from (2) and one item from (3) ${ }^{\mathrm{a}}$

Buitelaar and van de Gaag [10]

a The diagnostic rules were not used in the latent class analysis where each item was considered independently 
syndrome. We consider, however, that the constellation of symptoms identified by the MCDD criteria may be in fact helpful in delineating a subgroup of ASD individuals with higher burden of psychotic symptoms. We consider the identification of more homegeneous subgroups as the first and necessary step in improving our understanding of the relationship between psychosis and developmental disorders.

Based on the above, we employed Latent Class Analysis (LCA) [21, 22] to classify children with ASD on the basis of the MCDD criteria as they provide an evidence-based framework for exploring further whether psychotic symptoms cluster within specific ASD classes. We elected to study a unique cohort of patients admitted between 2003 and 2012 to a specialist children's inpatient unit based at South London and Maudsley NHS Foundation Trust which receives national referals for children with severe and complex developmental and neuropsychiatric disorders. The advantage of this cohort is that it complements community-based studies (a) by focusing on children with severe and complex phenomenology where differential diagnosis between ASD with psychotic symptoms and early onset schizophrenia is a recurring and challenging clinical problem, (b) because of the availability of detailed professional observations of children's behaviour which allows for the disambiguation of complex symptoms that is not always feasible based on parental or teacher reports. Our goals were (a) to specify the number and types of ASD classes according to MCDD criteria (b) to validate the derived classes on the basis of the presence of hallucinations, a psychotic symptom outside the MCDD criteria, and (c) to determine whether the identified classes differ in terms of their overall function and response during inpatient treatment.

\section{Methods}

Sample

The sample comprised all cases fulfilling ICD-10 criteria for ASD (http://apps.who.int/classifications/icd10/browse/ 2010/en) (categories F84.0-F84.9) at discharge from Acorn Lodge Unit, a national specialist inpatient service for children up to the age of 13 years. Cases of childhoodonset schizophrenia (ICD-10 F20) were excluded regardless of whether they also met criteria for ASD. No other exclusion criteria were applied. Diagnostic assessment was based on combinations of comprehensive developmental history, detailed observations during admission, the Autism Diagnostic Interview-Revised [23] and the Autism Diagnostic Observation Schedule [24]. All comorbid diagnoses were recorded. Global function was assessed using the Children's Global Assessment Scale scores (CGAS) [25] on admission and at discharge. Family history of mental illness in first-degree relatives, age on admission, medication type and dose, and length of inpatient stay were also recorded.

We used a structured proforma to record the presence or absence of each of the MCDD criteria (Table 1) based on the patients' case notes. Abnormal sensory phenomena were considered psychotic hallucinatory experiences if they were supported both by self-report and behavioural observations, were recurrent and present every day or nearly every day, were not exclusively related to the sleep/ wake cycle or to environmental circustances (e.g. following fights). The Child Psychiatry services at the South London and Maudsley NHS Foundation Trust use a structured format what included very detailed and comprehensive information regarding clinical presentation and treatment. The use of our case notes to derive clinically relevant information over long periods of observation, especially for disorders with low prevalence, has been validated in multiple previous studies notably in connection to early onset schizophrenia (e.g. [26, 27]). Twelve case notes were also rated independently by two qualified child psychiatrists (MR and SM) and showed high interrater reliability $(\kappa=0.72, p<0.001)$.

\section{Statistical analysis}

LCA of the indidiual MCDD criteria as binary items was performed to test whether it was possible to identify distinct classes of ASD children. Each MCDD criterion was recorded as present or absent as our intention was to use the MCDD criteria as the general framework for determining latent classes. It is important to note that LCA analyses did not depend on whether any particular case met the criteria for MCDD as a disorder. In other words, the rules for MCDD diagnosis (Table 1) were not applied for the LCA. LCA is a probabilistic modelling technique which can detect latent (i.e. not directly measured) homogeneous nonoverlapping classes underlying observed data [28]. A further advantage of the LCA compared to other cluster analyses is that it defines mutually exclusive classes using maximum likelihood estimation [29]. Entropy was used as a measure of classification uncertainty measured on a $0-1$ scale with a value of 1 indicating perfect classification [30]. Model fit was assessed by average latent class posterior probabilities for most likely class membership by latent class [31]. The optimal number of classes was determined by the Bayesian information criterion (BIC) [32]; smaller BIC values indicating overall model improvement $[31,33]$. In addition, the bootstrap likelihood ratio test (BLRT) was chosen as the best performing likelihood ratio test [33]. LCA was performed in MPlus Version 5 [34]. Finally, 
either Pearson's $\chi^{2}$ or Fisher's Exact Test (if cell count $<5$ ) was used to examine whether sex, hallucinations, comorbid diagnosis of depressive disorder (ICD-10 F32) or antipsychotic treatment differed between the LCA-identified latent classes. Student's $t$ test was used to compare LCA-identified latent classes in terms of CGAS and length of inpatient stay. Due to the positively skewed distribution in length of stay, square root transformation was additionally employed. Survival analysis was conducted to estimate time-to-discharge patterns in different groups.

The study was approved by the South London and Maudsley NHS Foundation Trust Child and Adolescent Mental Health Services Clinical Governance Committee under the service evaluation remit.

\section{Results}

Eighty-four patients with ASD (25\% female) and an average age of 11.1 years $(\mathrm{SD}=1.6)$ on admission were included in this study.

\section{Latent class analysis}

LCA analysis with the MCDD items supported a 2-class model over a 1-class and 3-class model on the basis of the BIC index (1-class solution: 864, 2-class solution: 847, 3-class solution: 861 ), the BLRT (1 class vs. 2 classes: $p<0.01,2$ classes vs. 3 classes: $p=0.33$ ) and Entropy ( 2 classes: 0.81, 3 classes: 0.89). Classification uncertainty rates for most likely class membership of the 2-class solution were very small $(0.077$ and 0.012$)$ indicating that there were no problems in class separation. As shown in Fig. 1, the two classes were significantly different in terms of peculiar and idiosyncratic fears and phobias, behavioural disorganisation, disordered and irrational thoughts, perplexity and overvalued paranoid ideas and fantasies. We designated the class showing high levels of affective and thought abnormalities as ASD with Psychotic features (ASD-P). The second class was designated ASD-NonP (without Psychotic features). This distinction was validated by the higher prevalence of hallucinations in the ASD-P group (26\%), compared to ASD-NonP (2.4\%) (Table 2). Of the children experiencing hallucinations, $92 \%$ were within ASD-P. Depressive and anxiety symptoms often cooccur in ASD children [14, 35] and therefore we compared the two classes in terms of the prevalence of depressive disorder (Table 2). Although no statistical difference was observed between the two classes, ASD-P had a numerically lower number of children with comorbid depression. Only nine children in the study sample had a first-degree relative affected with psychosis. Five had a first-degree relative with bipolar disorder and four with SZ. The small sample precludes formal statistical comparisons. Of note, all patients with a family history of bipolar disorder belonged to the ASD-NonP class, while 3 of the 4 patients

Latent Classes for Multiple Complex Developmental Disorder Scale Items

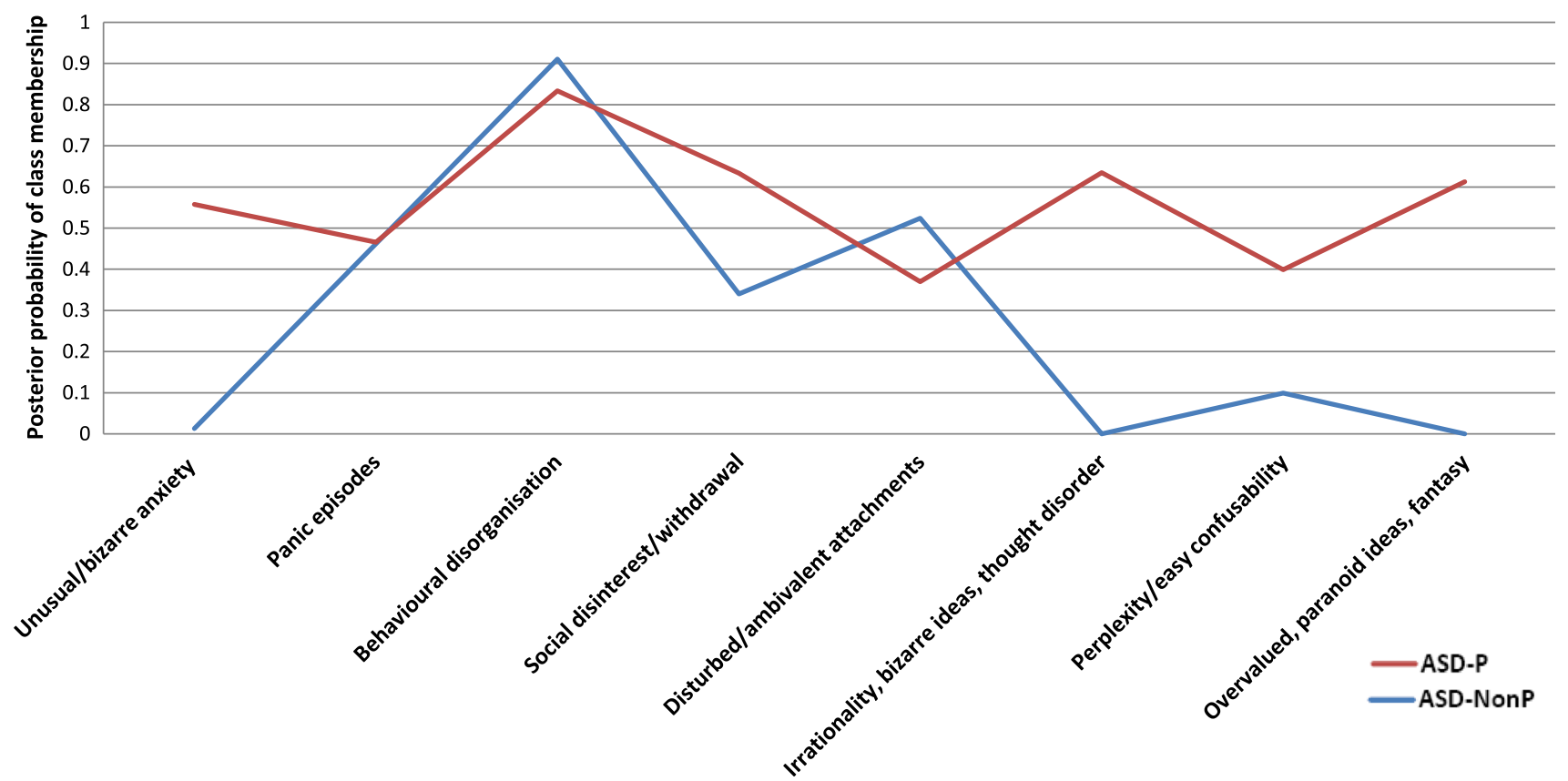

Fig. 1 Latent classes for multiple complex developmental disorder scale items 
Table 2 Sample demographic and clinical characteristics

\begin{tabular}{lccl}
\hline & ASD-P & ASD-NonP & $p$ \\
& $N=43$ & $N=41$ & \\
\hline Age on admission (years) & $11.30(1.68)$ & $10.84(1.59)$ & $\mathbf{0 . 2 0}$ \\
Female sex (\%) & $10(23)$ & $11(27)$ & $\mathbf{0 . 7 0}$ \\
Hallucinations (\%) & $11(26)$ & $1(2.4)$ & $\mathbf{0 . 0 0 2}$ \\
Comorbid depressive disorder & $4(9 \%)$ & $7(17 \%)$ & $\mathbf{0 . 2 9}$ \\
$\quad(\%)$ & & & \\
CGAS on admission & $25(12)$ & $28(11)$ & $\mathbf{0 . 2 8}$ \\
CGAS at discharge & $46(16)$ & $45(14)$ & $\mathbf{0 . 7 1}$ \\
Antipsychotic medication $(\%)$ & $23(53)$ & $20(49)$ & $\mathbf{0 . 6 6}$ \\
Length of Inpatient Stay & $151(78)$ & $115(63)$ & $\mathbf{0 . 0 2}$ \\
$\quad$ (days) & & & \\
\hline
\end{tabular}

Continuous data are shown as mean (standard deviation); Categorical data are shown as number $(\%)$

Significance group differences in bold typeface

$A S D$ Autism Spectrum Disorder, ASD-P ASD with Psychotic features, ASD-NonP ASD without Psychotic features, CGAS Children's global assessment scale

with a first-degree relative with psychosis belonged to the ASD-P class.

Global functioning and inpatient treatment

As shown in Table 2, the two classes did not differ in terms of antipsychotic treatment or global function on admission or discharge. However, the length of inpatient stay was prolonged on average by 36 days in the ASD-P compared to the ASD-NonP class and square root transformation did not affect these results. Survival analysis also identified different time-to-discharge plots between ASD-P and ASD-NonP $(z=4.29, d f=1, p=0.038)$ in the same direction (Fig. 2).

Relationship of the MCDD diagnosis to the latent classes

Although not a focal point of this study, our data allowed us to compare children that met diagnostic criteria for MCDD and those in the ASD-P and ASD-NonP latent classes. To this purpose, we used the MCDD scoring rules (Table 1) to identify those children that could be diagnosed as MCDD and we then examined their assignement to either of the two latent classes. Twenty-eight cases (20 male) fulfilled criteria for MCDD. Nearly all children with MCDD (26; $93 \%$ ) were assigned to the ASD-P class. Children with MCDD comprised $60 \%$ of the ASD-P class and $58 \%$ of the children that experienced hallucinations were within MCDD.

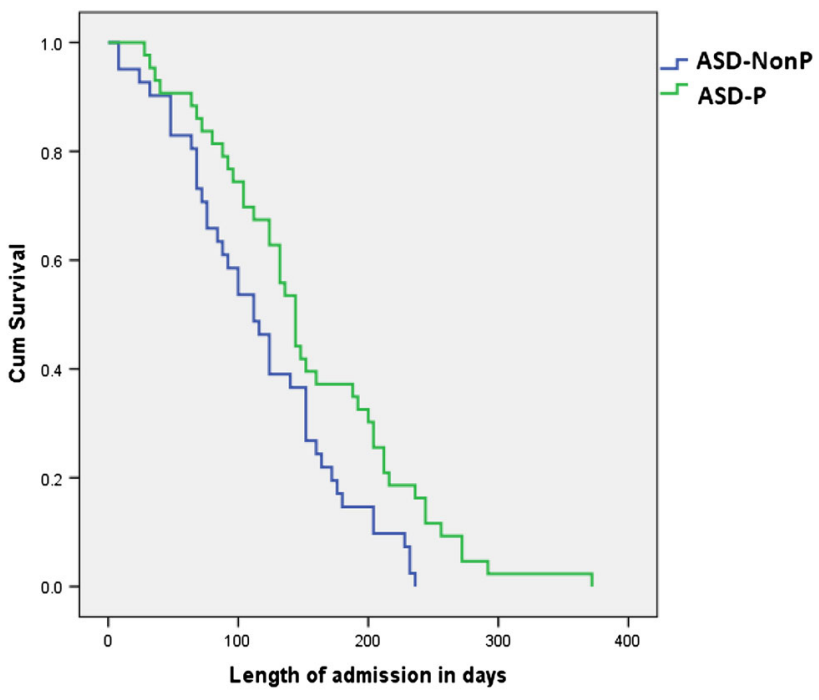

Fig. 2 Survival analysis: time-to-discharge plots comparing ASD-P with ASD-NonP $(z=4.29, d f=1, p=0.038)$. ASD Autism Spectrum Disorder, $A S D-P$ ASD with psychotic features, $A S D$-NonP ASD without psychotic features

\section{Discussion}

We found that LCA of the MCDD criteria resulted in two classes of ASD patients, one with (ASD-P) and one without (ASD-NonP) psychotic features. Children that met full criteria for MCDD represented a significant subset of the ASD-P class. Our results therefore confirm the usefulness of the MCDD concept in exploring the relationship between ASD and psychosis but suggest that MCDD as a diagnosis is probably too restrictive.

The identification of two classes of ASD children using LCA is a significant and novel finding because these classes were derived directly from data, rather than defined a priori based on theoretical assumptions. To our knowledge, this is the first empirically derived classification of ASD children in terms of clinical profiles related to psychosis. Consistent with previous literature we identified bizarre anxiety reactions/peculiar phobias and thought disorder as two key symptom domains associated with psychosis in ASD [12, 17]. This is particularly reassuring since the sample in this study cannot be considered representative in epidemiological terms. Our results, however, suggest that the association of idiosyncratic fears and thought disorder with psychosis is present regardless of sampling biases. Our study design does not allow us to comment on the neurobiological underpinnings of these observations. Our family history data are limited by sample size but hint to an over-representation of children with a first-degree relative with SZ in the ASD-P class.

It is noteworthy that affective psychopathology and thought disorder are emerging as key predictors of 
transition to overt psychosis in a wide range of high-risk groups $[12,20]$. Therefore, from the perspective of clinicians treating individuals with ASD, the presence of abnormalities in both these domains in a child with ASD is likely to result in psychotic experiences. The MCDD concept as a diagnostic entity may be restrictive, but the MCDD criteria highlight the importance of thought disorder and unusual or peculiar fears and phobias or frequent idiosyncratic or bizarre anxiety reactions in children with ASD and are particularly useful in defining an ASD subgroup more closely linked to psychosis.

On admission, children with ASD had very low CGAS scores (Table 2) indicative of inability to function in most situations. The ASD-P and ASD-NonP classes did not differ in this respect probably because of a floor effect. Both classes were able to benefit considerably by inpatient specialist intervention and at the point of discharge they presented with similar CGAS scores in the range of moderate impairement in most areas (Table 2). During the admission, both classes were equally likely to receive antipsychotics (Table 2). The ASD-P group remained in the unit for an average of 36 days longer than the ASDNonP group (Table 2). This finding opens a new direction of enquiry to define the reasons for the delayed discharge of the ASD-P class. Future studies on this issue may identify either social or treatment-related reasons for this delay and suggest ways to optimise the clinical care of this group.

Several methodological issues need to be considered. First, LCA analysis is a powerful analytical tool for the identification of more homegeneous classes of patients but it does not provide proof that these classes exist. This will require studies examining the reproducibility of our findings. Second, the sample size in this study is relatively small, which is inherent to the population under study, and the study's naturalistic design. The cross-sectional design also does not allow us to comment on the long-term clinical outcome of these children. Further investigations in significantly larger and independent samples are required to determine the prognostic value of our classification and their relationship to treatment response.Third, the variables entered in the LCA models were based on the MCDD concept. Other disease dimensions (e.g. genetic, brain structural and functional measures) may also be relevant and may be used to refine our classification in future studies.

\section{Conclusions}

In summary, we have shown that the MCDD criteria that emphasise unusual or peculiar fears and phobias or frequent idiosyncratic or bizarre anxiety reactions and thought disorder are useful in identifying a class of children with ASD more likely to present with psychosis. Future studies should focus on validating these results and determining optimal interventions for ASD children with psychotic features.

Conflict of interest On behalf of all authors, the corresponding author states that there is no conflict of interest.

\section{References}

1. Cheung C, Yu K, Fung G, Leung M, Wong C, Li Q, Sham P, Chua S, McAlonan G (2010) Autistic disorders and schizophrenia: related or remote? An anatomical likelihood estimation. PLoS One 5:e12233

2. Sugranyes G, Kyriakopoulos M, Corrigall R, Taylor E, Frangou S (2011) Autism spectrum disorders and schizophrenia: meta-analysis of the neural correlates of social cognition. PLoS One 6:e25322

3. Crespi B, Stead P, Elliot M (2010) Evolution in health and medicine Sackler colloquium: comparative genomics of autism and schizophrenia. Proc Natl Acad Sci USA 107:1736-1741

4. Rapoport J, Chavez A, Greenstein D, Addington A, Gogtay N (2009) Autism spectrum disorders and childhood-onset schizophrenia: clinical and biological contributions to a relation revisited. J Am Acad Child Adolesc Psychiatry 48:10-18

5. Sullivan PF, Magnusson C, Reichenberg A, Boman M, Dalman C, Davidson M, Fruchter E, Hultman CM, Lundberg M, Långström N, Weiser M, Svensson AC, Lichtenstein P (2012) Family history of schizophrenia and bipolar disorder as risk factors for autism. Arch Gen Psychiatry 69:1099-1103

6. Bevan Jones R, Thapar A, Lewis G, Zammit S (2012) The association between early autistic traits and psychotic experiences in adolescence. Psychiatr Res 135:164-169

7. Mouridsen Rich, Isager J, Nedergaard NJ (2008) Psychiatric disorders in individuals diagnosed with infantile autism as children: a case control study. Psychiatr Pract 14:5-12

8. Unenge Hallerbäck M, Lugnegård T, Gillberg C (2007) Is autism spectrum disorder common in schizophrenia? Psychiatry Res 198:12-17

9. Solomon M, Ozonoff S, Carter C, Caplan R (2008) Formal thought disorder and the autism spectrum: relationship with symptoms, executive control, and anxiety. J Autism Dev Disord 38:1474-1484

10. Buitelaar JK, Van der Gaag RJ (1998) Diagnostic rules for children with PDD-NOS and multiple complex developmental disorder. J Child Psychol Psychiatry 39:911-919

11. Van der Gaag RJ, Buitelaar J, Van den Ban E, Bezemer M, Njio L, Van Engeland H (1995) A controlled multivariate chart review of multiple complex developmental disorder. J Am Acad Child Adolesc Psychiatry 34:1096-1106

12. Sprong M, Becker HE, Schothorst PF, Swaab H, Ziermans TB, Dingemans PM, Linszen D, van Engeland H (2008) Pathways to psychosis: a comparison of the pervasive developmental disorder subtype Multiple Complex Developmental Disorder and the "At Risk Mental State”. Schizophr Res 99:38-47

13. Kanner L (1943) Autistic disturbances of affective contact. Nervous Child 2:217-250

14. Simonoff E, Pickles A, Charman T, Chandler S, Loucas T, Baird G (2008) Psychiatric disorders in children with autism spectrum disorders: prevalence, comorbidity, and associated factors in a population-derived sample. J Am Acad Child Adolesc Psychiatry 47:921-929 
15. van Steensel FJ, Bögels SM, Perrin S (2011) Anxiety disorders in children and adolescents with autistic spectrum disorders: a metaanalysis. Clin Child Fam Psychol Rev 14:302-317

16. White SW, Oswald D, Ollendick T, Scahill L (2009) Anxiety in children and adolescents with autism spectrum disorders. Clin Psychol Rev 29:216-229

17. Weisbrot DM, Gadow KD, DeVincent CJ, Pomeroy J (2005) The presentation of anxiety in children with pervasive developmental disorders. J Child Adolesc Psychopharmacol 15:477-496

18. van der Gaag RJ, Caplan R, van Engeland H, Loman F, Buitelaar JK (2005) A controlled study of formal thought disorder in children with autism and multiple complex developmental disorders. J Child Adolesc Psychopharmacol 15:465-476

19. Sporn AL, Addington AM, Gogtay N, Ordoñez AE, Gornick M, Clasen L, Greenstein D, Tossell JW, Gochman P, Lenane M, Sharp WS, Straub RE, Rapoport JL (2004) Pervasive developmental disorder and childhood-onset schizophrenia: comorbid disorder or a phenotypic variant of a very early onset illness? Biol Psychiatry 55:989-994

20. Thompson A, Nelson B, Bruxner A, O'Connor K, Mossaheb N, Simmons MB, Yung A (2013) Does specific psychopathology predict development of psychosis in ultra high-risk (UHR) patients? Aust N Z J Psychiatry 47:380-390

21. Stahl D, Sallis H (2012) Model-based cluster analysis. WIREs. Comput Statistics 4:341-358

22. Stringaris A, Stahl D, Santosh P, Goodman R (2011) Dimensions and latent classes of episodic mania-like symptoms in youth: an empirical enquiry. J Abnorm Child Psychol 39:925-937

23. Lord C, Rutter M, Goode S, Heemsbergen J, Jordan H, Mawhood L, Schopler E (1989) Autism diagnostic observation schedule: a standardized observation of communicative and social behavior. J Autism Dev Disord 19:185-212

24. Lord C, Rutter M, Le Couteur A (1994) Autism Diagnostic Interview-Revised: a revised version of a diagnostic interview for caregivers of individuals with possible pervasive developmental disorders. J Autism Dev Disord 24:659-685
25. Shaffer D, Gould MS, Brasic J, Ambrosini P, Fisher P, Bird H, Aluwahlia S (1983) A children's global assessment scale (CGAS). Arch Gen Psychiatry 40:1228-1231

26. Hollis C (2003) Developmental precursors of child- and adolescent-onset schizophrenia and affective psychoses: diagnostic specificity and continuity with symptom dimensions. Br J Psychiatry $182: 37-44$

27. Cannon M, Walsh E, Hollis C, Kargin M, Taylor E, Murray RM, Jones PB (2001) Predictors of later schizophrenia and affective psychosis among attendees at a child psychiatry department. Br J Psychiatry 178:420-426

28. Muthen B (2001) Latent variable mixture modeling. In: Marcoulides GA, Schumaker RE (eds) New developments and techniques in structural equation modeling. Lawrence Erlbaum Associates, Hillsdale

29. Agresti A (2002) Categorical data analysis. Wiley Interscience, Hoboken

30. Celeux G, Soromenho G (1996) An entropy criterion for assessing the number of clusters in a mixture model. J Classif 13:195-212

31. Hagenaars JA, McCutcheon AL (2002) Applied Latent Class Analysis. Cambridge University Press, Cambridge, p 454

32. Schwarz G (1978) Estimating the dimension of a model. The Annals of Statistics 6:461-464

33. Nylund KL, Asparouhov T, Muthen BO (2007) Deciding on the number of classes in latent class analysis and growth mixture modeling: a monte carlo simulation study. Struct Equ Model 14:535-569

34. Muthen LK, Muthen BO (2007) Mplust User's guide, 5th edn. Muthen \& Muthen, Los Angeles

35. Strang JF, Kenworthy L, Daniolos P, Case L, Wills MC, Martin A, Wallace GL (2012) Depression and anxiety symptoms in children and adolescents with autism spectrum disorders without intellectual disability. Res Autism Spectr Disord 6:406-412 